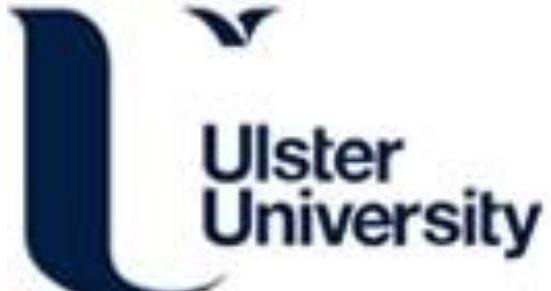

\section{The Use of Gamification Techniques in a Clinical Setting for the Collection of Longitudinal Kinematic Data}

Ennis, A., Cleland, I., Nugent, CD., Finney, L., Trainor, D., \& Bennett, A. (2016). The Use of Gamification Techniques in a Clinical Setting for the Collection of Longitudinal Kinematic Data. In The Use of Gamification Techniques in a Clinical Setting for the Collection of Longitudinal Kinematic Data (pp. 267-273). SPRINGER LINK. https://doi.org/10.1007/978-3-319-48746-5_27

Link to publication record in Ulster University Research Portal

\section{Published in:}

The Use of Gamification Techniques in a Clinical Setting for the Collection of Longitudinal Kinematic Data

Publication Status:

Published (in print/issue): 02/11/2016

DOI:

https://doi.org/10.1007/978-3-319-48746-5_27

\section{Document Version}

Author Accepted version

\section{General rights}

Copyright for the publications made accessible via Ulster University's Research Portal is retained by the author(s) and / or other copyright owners and it is a condition of accessing these publications that users recognise and abide by the legal requirements associated with these rights.

\section{Take down policy}

The Research Portal is Ulster University's institutional repository that provides access to Ulster's research outputs. Every effort has been made to ensure that content in the Research Portal does not infringe any person's rights, or applicable UK laws. If you discover content in the Research Portal that you believe breaches copyright or violates any law, please contact pure-support@ulster.ac.uk. 


\title{
The Use Of Gamification Techniques In A Clinical Setting For The Collection Of Longitudinal Kinematic Data
}

\author{
Andrew Ennis ${ }^{1}$, Ian Cleland ${ }^{1}$, Chris Nugent ${ }^{1}$, Laura Finney ${ }^{2}$, David Trainor ${ }^{3}$, Aidan \\ Bennett ${ }^{4}$.
${ }^{1}$ Computer Science Research Institute and School of Computing and Mathematics, University of Ulster, Newtownabbey, Co. Antrim, Northern Ireland, BT37 0QB
\{a.ennis, i. cleland, cd.nugent\}@ulster.ac.uk
${ }^{2}$ Leckey, 19C Ballinderry Road, Lisburn, Northern Ireland, BT28 2SA. laura.finney@leckey.com Queens Island, Belfast, Northern Ireland, BT3 9DT. david.trainor@sentireal.com. \\ ${ }^{3}$ Sentireal, Queens University of Belfast Northern Ireland Science Park, Queens Road, \\ ${ }^{4}$ Data Analytics Labs Ltd., Scottish Provident Building, 7 Donegall Square West, Belfast, \\ Northern Ireland, BT1 6JH. \\ aidanbennett@dataanalyticslabs.com.
}

\begin{abstract}
Children with physical impairments, ranging from impaired mobility to very limited mobility, often require mobility aids to compensate for these difficulties. These impairments can adversely affect the child to varying degrees and have an impact on their health and wellbeing. It is estimated that $30 \%-40 \%$ of medical interventions have no reported evidence base and another $20 \%$ of interventions delivered are ineffective. Clinicians are under increasing pressure to provide evidence of the effectiveness of prescribed treatments and products. Therefor there is a need to provide clinicians with empirical data that evidences practice and provides a quantified assessment of treatment efficacy through data gathering in both real-time and longitudinally, combined with data analytics to further develop treatment strategies. This paper presents a system to assist and enable clinicians to analyze and asses the effectiveness and usage of prescribed treatments for physically impaired children. The system achieves this through the use of a gamified data collection app and a web portal to analyze and present summarized measures of gait.
\end{abstract}

Keywords: Gamification, Step Counting, Postural Aids, Mobility.

\section{Introduction}

Children with physical impairments may suffer from limitations in walking, ranging from impaired to no walking. Often they require mobility aids or wheelchairs to compensate for these difficulties [1]. Physical impairments can to varying degrees, adversely affect the physical, cognitive and sensory development of a child, with adverse impacts on health and wellbeing [1]. These impacts can be influenced by a number of factors, such as; body structure and function, daily activities of personal care, 
participation in home, school, work and family issues and other personal factors [1], [2]. Reductions in muscular strength, difficulties in coordination, and alterations in the gross and fine motor movements are the most common limitations caused by physical motor disabilities [3]. On many occasions, limitations of motor impairments require the use of assistive devices, such as wheelchairs and postural aids and these form a large part of standard care for children with physical disabilities[4]. Such devices can increase a child's independence, participation and quality of life by improving their mobility [5]. It is estimated that $30 \%-40 \%$ of medical interventions have no reported evidence base and another $20 \%$ of interventions delivered are ineffective, unnecessary, or cause harm [4]. Currently within the pediatric rehabilitation sector clinicians are under increasing pressure to provide evidence of the effectiveness of treatments and products prescribed for children they are referred. Due to the nature of the pediatric special needs market there is little published research into the effectiveness of mobility and postural aids [4]. Clinicians are under increasing pressure to provide this evidence, however, the role of a clinician is to diagnose and assess impairment in the patient, not the performance of the mechanism or device that delivers this improvement. Nevertheless, by increasing knowledge in the areas of intervention and clinical research the potential exists to achieve system-wide improvements in health care quality and health outcomes. There is therefore a real need to provide clinicians with empirical data that evidences practice [4]. Therefore a quantitative way of assessing treatment efficacy, through data gathered in real-time and collated longitudinally, combined with data analytics to provide summary information to clinicians, parents and users is essential to manage and further develop treatment strategies [6].

The most commonly used devices to improve the standing position of children are mobility supports and adaptive seating, that provide more autonomy and diminish dependence [3]. Most of the research studies evaluating gait have focused on gross motor skills and analysis of the degree of movement, however, have not examined the quality of movement in terms of kinematics and geometry of the movement [3].

\section{Implementation}

We consulted with three experts in pediatric physiotherapy who provided us with an insight into the clinical needs of treating a child with walking impairments. With this clinical expertise, combined with a review of the literature, it was possible to identify areas on which to focus the platform. These namely being a gamified data collection app, based on the detection of step counts and a web portal aimed at clinicians to allow the review of the child's progress and clinical parameters of gait.

In order to facilitate the data gathering and presentation, we developed a platform that enabled the collection of accelerometer data, which is uploaded to a cloud backend. This allows clinicians to view, analyze and summarize the data. The collected and analyzed data will allow a clinician to better determine the effectiveness of a given treatment. This was highlighted as an issue in both the literature review and engagement with clinicians. We gamified the data collection app in order to improve engagement of the child with the solution which in turn increases the amount of accelerometer data generated by the child. The mobile app connects to a Shimmer (Shimmer 3, Shimmer 
research, Dublin) device via Bluetooth, as shown in Figure 1A, to collect the accelerometer data. Shimmer is a well know platform within the connected health and assisted living research domains and offers a range of onboard sensing technologies, such as 3-axis accelerometer, digital magnetometer, gyro and pressure sensor [7], [8]. The Shimmer platform also has the capability of optional expansion modules that enable ECG, EMG and GSR [7]. When in use, the Shimmer device is attached to the child, either on the feet or lower back, or it may be integrated within the walker itself.

The mobile app was developed on top of UWP (Universal Windows Platform) in $\mathrm{CH}$. This allowed the app to be deployed to a wide range of computing devices, ranging from a smartphone to Raspberry Pi 3, or laptop/desktop computer. Figure 1A shows the app running on a Windows mobile. The system architecture can be seen in Figure 1B and the web portal running in a smartphone browser can be seen in Figure 1C.

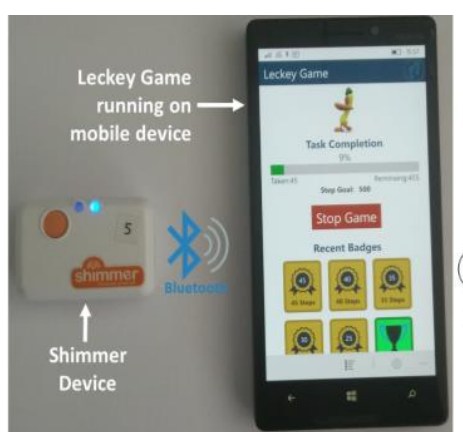

(A)

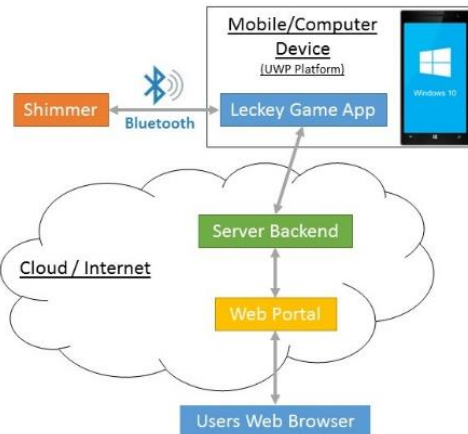

(B)

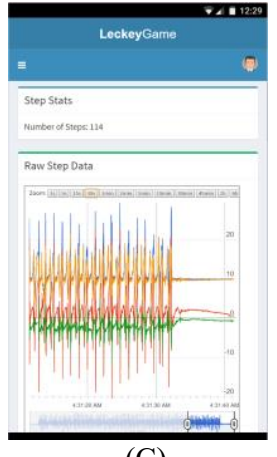

(C)

Fig. 1. (A) Shimmer device and Leckey Game app running on mobile phone.

(B) System Architecture. (C) Web Portal displayed in web browser of a mobile smart phone.

\subsection{Gamification}

In order to gamify the app, we selected step counts as the metric used for the game, because steps are a commonly used metric within health promotion[9]. The objective of the game is to complete a set amount of steps per day, which is determined by the clinician. Currently, for testing purposes, we have the step achievement badges set at 5 step increments. This can, however, be set by the clinician to give a personalized target and will vary depending on the child's abilities. When an achievement badge is unlocked a notification is displayed to the user to alert them and encourage them to keep progressing. A new achievement badge is then displayed in the "Recent Badges" section, as can be seen on the mobile device in Figure 1A.

\subsection{Step Counting Algorithm}

For the gamification of the app, it is necessary to calculate step counts locally on the device, in real-time. This also enables the game to run on the mobile device without the need to connect to external services and therefore does not consume mobile data. 
An example of two walking steps of the left foot, from an un-impaired adult is shown in figure 3. The first step in the figure has been annotated to show the various points of the foot step.

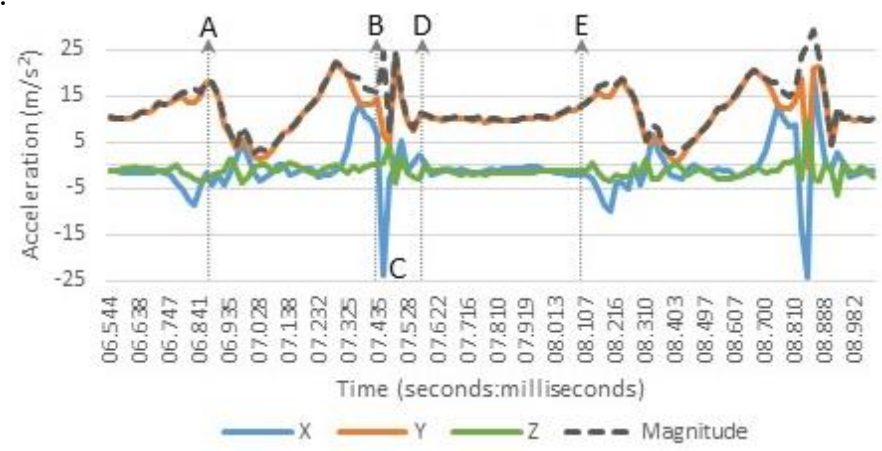

Fig. 3. Graph showing accelerometer data of 2 steps of the left foot, of an un-impaired adult. A: toe off. A to B: foot swing. C: heal strike. D to E: Left foot stationary, right foot moving.

To detect a step, we use an algorithm that analyses the $x, y, z$ from the 3 -axis accelerometer data to determine if the accelerations match a step profile. The algorithm we use is based on the algorithm proposed by [10] which detects peaks that are above a dynamic threshold. The first step in the algorithm is to calculate the magnitude for every $\mathrm{x}, \mathrm{y}, \mathrm{z}$ data point, as shown in the equation below. This means the Shimmer device can be orientation free and still reliably detect steps.

$$
\text { Mag }=x^{\wedge} 2+y^{\wedge} 2+z^{\wedge} 2
$$

We then take a sliding window, containing 50 samples, and calculate the maximum, minimum and average, of the sliding window, and continuously update them every 50 samples, as shown in Figure 4. The average is used as the dynamic threshold and is dynamic because it is updated every 50 samples.

We define that a step has been taken when a new magnitude point is less than the old magnitude point, e.g. a negative slope, when it crosses the threshold. Figure 4 shows a sample of step data from an un-impaired adult, along with the calculated maximum, minimum and dynamic threshold.

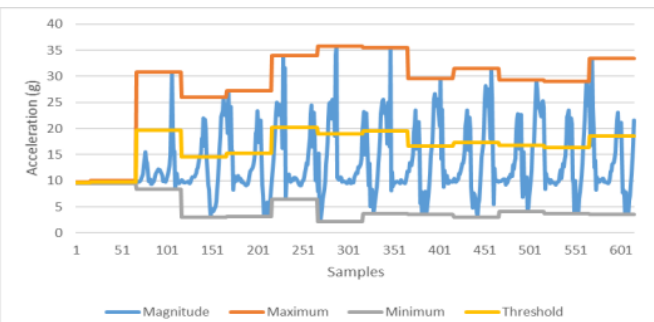

Fig. 4. Graph showing magnitude and the calculated max, min of the sliding window, updated every 50 samples. The graph also shows the calculated threshold of the sliding window, updated every 50 samples. 


\section{Results}

Initial testing of the step counting algorithm and data collection app was carried out on two un-impaired adults from the research team without a postural aid and then subsequently while using a postural aid (enlarged for adults and built by Leckey). The test consisted of wearing the Shimmer device on the left outer ankle and walking around the perimeter of a defined rectangle. The size was determined by the limited open area available to test in. the participant was instructed to place the shimmer device in any orientation and this was not constrained. The participants walked 100 steps of the left foot. This was counted with a tally counter by an observer and the observer stopped the participant once 100 steps was achieved. Our reason for selecting 100 steps, is that a child with impaired walking is likely to take less steps than a non-impaired child. Hence our algorithm must detect as many of the steps as possible to be a viable metric for gamification purposes.

When 100 steps were taken without the postural aid, both participants' step counts were very close, being only 2 steps over the ground truth measured by the observer, as shown in Table 1. Nevertheless, when using the postural aid, the number of step counts significantly varied from the ground truth (Table 1). For participant 1 this was up to 25 steps over the ground truth. Participant 2 was closer to the ground truth, being only 5 steps over the ground truth.

Table 1. Two evaluations from two participant for both no postural aid and with a postural aid. $\mathrm{P} 1$ being participent 1 and $\mathrm{P} 2$ being participant 2.

\begin{tabular}{lccc}
\hline & Ground Truth & Evaluation 1 & Evaluation 2 \\
\hline P1 - No Postural Aid & 100 & 101 & 102 \\
P2 - No Postural Aid & 100 & 101 & 100 \\
P1 - With Postural Aid & 100 & 116 & 125 \\
P2 - With Postural Aid & 100 & 101 & 105 \\
\hline
\end{tabular}

Figure 5 shows the first 5 seconds of step accelerometer data without a postural aid (A) and with a postural aid (B). It is evident from the Figure that the step pattern is similar, however, with the postural aid (B) the steps are less pronounced and have a weaker magnitude.

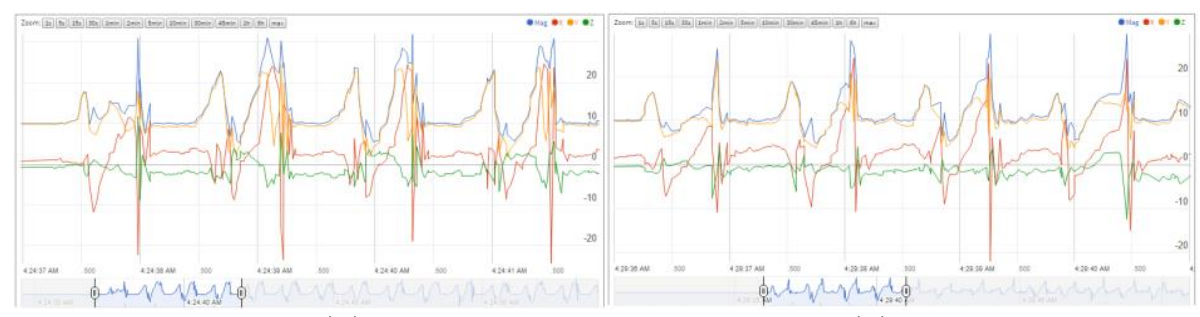

(A)

(B)

Fig. 5. Graph A showing first 5 seconds of unfiltered walking data without a postural aid. Graph B shows first 5 seconds of unfiltered walking data with a postural aid, from the same participant with the Shimmer located in the same position and orientation. 


\section{Conclusions}

The results demonstrate that, when the postural aid was used the algorithm over counted the number of steps. This is because the accelerometer magnitude was less pronounced and therefore the dynamic threshold was lower, hence more minor step movements were counted as a step. Based on these results it appears the step counting algorithm is too sensitive when dealing with less defined footsteps. Due to this issue a normal step counting algorithm will not work very accurately. A solution maybe to independently run step detection on each axis, along with the magnitude and then using an assigned weighting on each axis to determine if a step has been taken. The weightings will be dynamically applied based on the amount of acceleration change during the sliding window. The step detection algorithm can also be modified to detect both peaks of the footstep profile and not just the heal strike, signifying the end of the step. As step count is only used for gamifying in the data collection app, being out by several steps is not critical, as the raw 3 -axis accelerometer data is what is critical to the clinician. This is analyzed by our backend system to determine other data metrics to provide an information summary to the clinician.

Future work will involve a larger study with child participants to better determine the accuracy of the step counting algorithm for children. We will also further develop the web portal to implement more summary information of gait (cadence, cycle times, stride length, trunk control and balance) to enable clinicians to better understand the usage and effectiveness of the prescribed treatment.

Acknowledgments. Invest Northern Ireland is acknowledged for supporting this project under the Competence Centre Programs Grant RD0513853 - Connected Health Innovation Centre.

\section{References}

[1] J. Casey, L. McKeown, R. McDonald, and S. Martin, "Wheelchairs for children under 12 with physical impairments," Cochrane Libr., no. 10, 2012.

[2] International Classification of Functioning, Disability, and Health: Children \& Youth Version: $I C F-C Y$. World Health Organization, 2007.

[3] S. M. Montero and a Gómez-Conesa, "Technical devices in children with motor disabilities: a review.," Disabil. Rehabil. Assist. Technol., vol. 9, no. 1, pp. 3-11, 2014.

[4] I. novak, S. Mcintyre, C. Morgan, L. Campbell, L. Dark, N. Morton, E. Stumbles, S. A. Wilson, and S. Goldsmith, "A systematic review of interventions for children with cerebral palsy: State of the evidence," Dev. Med. Child Neurol., vol. 55, no. 10, pp. 885-910, 2013.

[5] M. Gough, "Continuous postural management and the prevention of deformity in children with cerebral palsy: An appraisal," Dev. Med. Child Neurol., vol. 51, no. 2, pp. 105-110, 2009.

[6] S. Patel, H. Park, P. Bonato, L. Chan, and M. Rodgers, "A review of wearable sensors and systems with application in rehabilitation.," J. Neuroeng. Rehabil., vol. 9, no. 12, pp. 1-17, 2012.

[7] Shimmer, "Shimmer3." [Online]. Available: http://www.shimmersensing.com/shop/shimmer3. [Accessed: 06-Jun-2016].

[8] A. Burns, B. R. Greene, M. J. McGrath, T. J. O’Shea, B. Kuris, S. M. Ayer, F. Stroiescu, and V. Cionca, "SHIMMER - A wireless sensor platform for noninvasive biomedical research," IEEE Sens. J., vol. 10, no. 9, pp. 1527-1534, 2010.

[9] C. Maher, A. Crettenden, K. Evans, M. Thiessen, M. Toohey, and J. Dollman, “A pedometer based physical activity self-management program for children and adolescents with physical disability design and methods of the StepUp study.," BMC Pediatr., vol. 14, p. 31, 2014.

[10] N. Zhao, "Full-featured pedometer design realized with 3-Axis digital accelerometer," Analog Dialogue, pp. 1-5, 2010. 